\title{
A self-similar model for shear flows in dense granular materials
}

\author{
G. Debregeas* and C. Josserand ${ }^{\dagger}$ \\ The James Franck Institute, The University of Chicago, \\ 5640 South Ellis Avenue, \\ Chicago, Illinois 60637, USA
}

\begin{abstract}
We propose a model to describe the quasistatic shearing of dry granular materials, which notably captures the differences in velocity profiles recently observed in 2 and $3-\mathrm{D}$ Couette flow experiments. In our scheme, the steady-state flow is due to the intermittent motion of particle clusters moving together with the wall. The motion of a cluster is associated with the transient formation of a fracture inside the sheared pack. The model is based on the existence of a persistence length for the fractures, which imposes a selfsimilar structure on the clusters. Through a probabilistic approach, we can evaluate the rate of appearance of a cluster of a given size and obtain a prediction for the average velocity profiles. We also predict the existence of large stress fluctuations at the moving wall, which characteristics are in good agreement with experimental data.
\end{abstract}

\section{INTRODUCTION}

Dry granular materials exhibit a large range of dynamical behaviours (for a general review see [1]). An assembly of non-cohesive macroscopic particles confined in a box can be considered as a solid, but will start flowing when submitted to a large enough shear stress. However, the underlying mechanism can be very different, depending on the shearing rate imposed and the resulting density of the material. Upon rapid shearing or shaking, a fluidized state is reached in which the stress is transfered from the boundaries to the bulk through binary collisions. This dynamics is well understood under the scope of the so-called kinetic theory [2]. In many practical situations however, gravity maintains a high density in the material so that the stress is mainly transfered by rubbing friction between particles in persistent contact. In this regime, all relative motion between particles tend to be confined to very narrow regions of the material, making a continuous approach inadequate. Within these so-called shear band, each particle is submitted to large forces which rapidly fluctuate as the packing environment and the associated force network evolve. The flow is driven by collective and jerky moves of large sets of particles, hold together by transient force chains. Although this regime is relevant to soil mechanics and geology (earthquakes and pyroclastic flows for example [3]) and important in industrial applications (granular transport in hoppers and pipes), a satisfactory description is to date lacking for such fully developed dense flows 佨.

A strong experimental effort has however been seen recently to probe the grain-scale dynamics within these shear bands, which now provides a good test for physical models: two series of experiments have been performed independently on 2-D and 3-D Couette cells. A collection of particles is confined between a fixed outer cylinder and a rotating inner one. In the 2-D experiment, the system consists of one monolayer of disc-like particles squeezed between 2 horizontal plates. In this geometry, a shear band is always present at the vicinity of the rotating inner cylinder. By different techniques, the authors were able to precisely measure the decay of the average particle velocity from the inner moving wall to the immobile region towards the outer cylinder [5.6]. Surprisingly enough, the two results were found to be significantly different: in 2-D the velocity $V$ decayed with the distance $r$ to the inner wall according to an exponential law, whereas in 3-D, the velocity profile was well fitted by a Gaussian centered on the surface of the wall. These results showed to be robust for polydisperse or highly irregularly shaped particles. For round and monodisperse grains however, a layering effect and a strong slippage between adjacent layers were observed which result in more complicated profiles.

In this note we will focus on the Gaussian/exponential behaviours as the spatial dimension changes. Because a Gaussian form cannot be derived from a local differential equation (for example see [7]), the 3-D velocity profile suggests that a purely local model would fail to describe it. Here we introduce non-local effects by postulating a correlation length in the particles displacements which depends on their position within the shear band. This approach comes to modeling the flow as a succession of transient fractures allowing the coherent motion of clusters of various sizes. This hypothesis alone allows us to produce an

\footnotetext{
* present address: Institut Charles Sadron, CNRS UPR 22, 6, rue Boussingault, 67083 Strasbourg Cédex

${ }^{\dagger}$ present address: Laboratoire de Modélisation en Mécanique, CNRS UMR 7607, Université Pierre et Marie Curie, Case 162, 8 Rue du Capitaine Scott, 75015 Paris-France
} 
average velocity profile consistent with what was observed in both 2- and 3-D. Finally, we will show that the probability distribution and the force spectrum derived from this model are in good agreement with experimental results found in the literature.

\section{THE MODEL}

We consider a plane shear geometry: a wall is moving at a constant speed $V_{0}$ along a half-space of granular material. The flow velocity is assumed to vanish far from the moving wall (see figure 1). We impose a no slip condition at the wall so that $V(0)=V_{0}$ (this boundary condition mimics a classical experimental realization where the first layer of particles is glued onto the moving wall). In our scheme, the motion of each particle can be decomposed into successive steps of typical length $d_{g}$ occurring at velocity $v=V_{0}$. Between two steps, the particle remains immobile. One grain can thus either be at rest or move together with the wall [ The interface between moving and static particles defines a failure surface for the pack. We define $P(r)$ as the stationary probability of a failure surface to be present, per unit of time and length (in units of particle diameter) along the $y$-axis, at a distance $r$ from the moving wall (as in ref [9]). Although a reduction in granular density by roughly $10 \%$ - the so-called Reynolds dilatancy - is known to accompany shear flow, we will first consider a uniform density. Both two and three dimensional geometries will be addressed; $D$ will denote in the following the space dimension. A 'fracture' will either be a line in 2-D, or a plane in 3-D.

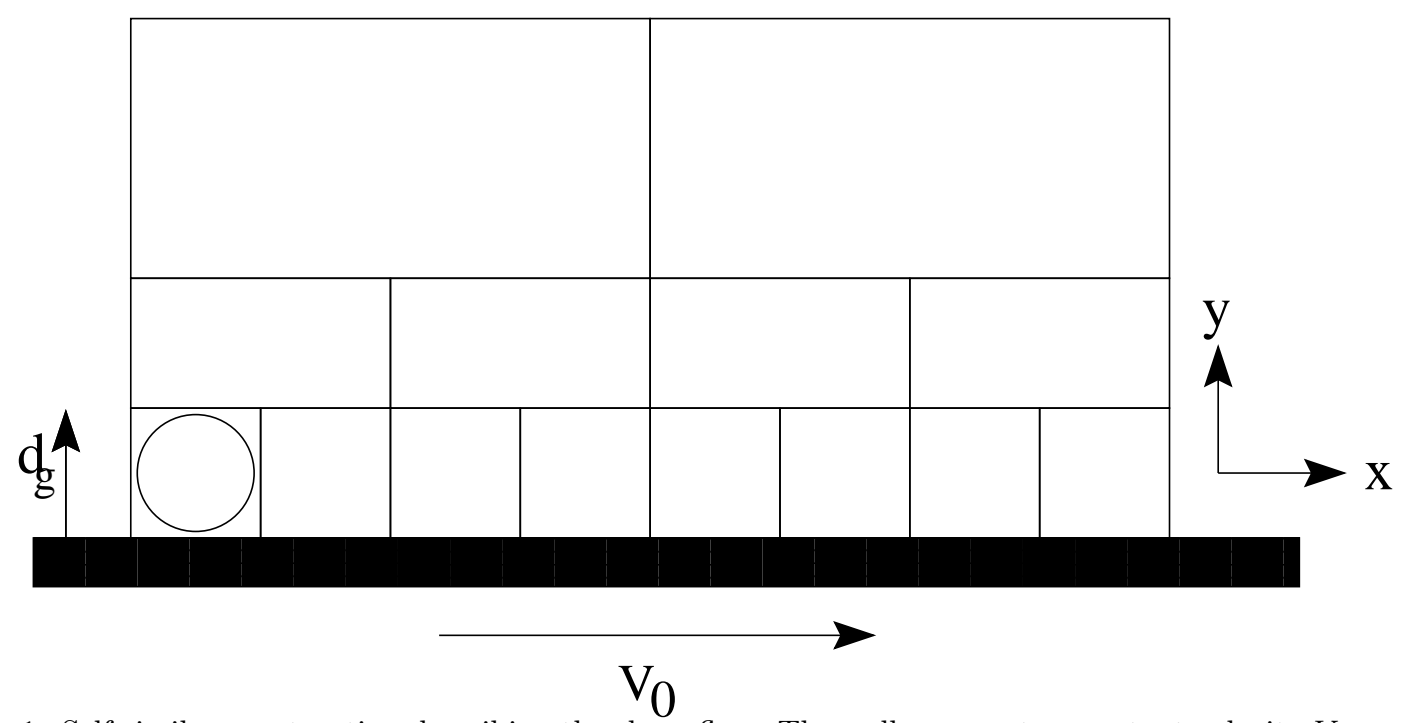

FIG. 1. Self-similar construction describing the shear flow. The wall moves at a constant velocity $V_{0}$ as well as the first layer of particles. At a distance $r$ from the wall, the flow is due to the sudden motion of clusters of size $r$ (or bigger) in all directions. The lines represent the possible location of fractures that appear in the deforming pack during the shearing process.

We first consider the dynamics of the first freely moving layer in the region $d_{g}<r<2 d_{g}$. As the wall moves by a distance $d_{g}$ in a time $\tau_{0}=d_{g} / V_{0}$, a particle located in this layer is either dragged by the wall producing a failure further away, or stays immobile so that a crack develops at exactly $r=d_{g}$. Assuming a Coulomb-type slip condition, the probability of the latter depends on the ratio of the normal and shear stress to which is submitted the particle during the time period $\tau_{0}$. Based on this mechanical consideration, and by analogy with thermally activated processes, Pouliquen \& al [9] have proposed an expression for the probability $p_{0}$ of slippage between 2 particles from 2 consecutive layers in a granular material slowly flowing down a pipe. In their picture, the spatial stress fluctuation induced by the randomness of the packing structure plays the role of a temperature, allowing them to apply classical rate processes theory [10]. In the quasi-static regime we address here, the average shear and normal stress are uniform in the material since each layer is constantly in mechanical equilibrium. In the limit of uniform density, it is therefore natural to assume a uniform value for $p_{0}$. Although, the

\footnotetext{
${ }^{1}$ note, this bimodal velocity distribution for the grains have been roughly observed in experiments [8]
} 
derivation of the slippage rate not only requires the knowledge of the static probability $p_{0}$. A typical fluctuation time-scale, related to the flow itself also needs to be introduced. This time-scale corresponds to the relaxation time of the stress network around the chosen particle. For a particle in the first layer, the only relevant time-scale is $\tau_{0}$ which is the time needed for a shear strain of 1 to be established. We can now write down the rate of slippage at the wall $P\left(d_{g}\right)$ as :

$$
P\left(d_{g}\right) \cdot d t \cdot \frac{d r}{d_{g}}=\mathcal{A} \cdot p_{0} \cdot \frac{V_{0} \cdot d t}{d_{g}} \cdot \frac{d r}{d_{g}}
$$

$\mathcal{A}$ is a constant introduced for normalization. It should be noted that we implicitly suppose a clear separation between the successive hopping events or equivalently that $p_{0}<<1$.

The calculation of $P(r)$ away from the wall is based on a self-similar argument. We postulate that, for a yielding event to occur at a distance $r$ from the wall, the crack must extend radially over a length of the order $r$. Hence the shearing of the material at a distance $r$ from the wall requires the coherent motion of a solid cluster of size $r$ in all directions. This cluster is dragged by the wall over a distance $d_{g}$ as one solid object, in the same way as was the single particle from the first layer (see figure 1). This self-similar description of the cluster size is introduced to account for the perturbation of the force network in the vicinity of the wall. Chain forces, which are responsible for the rigidity of the dense pack, are screened by the proximity of a solid boundary. Independent motion of particles are thus only allowed in the vicinity of the wall whereas the bulk behaves as a solid body. We note that an identical scaling have been successfully postulated in (at least) 2 very different contexts to describe the effect of a wall on dynamical structures. To estimate the Prandtl mixing layer in turbulent flows near a wall, the characteristic length of the vortices are assumed to increase like the distance to the boundary layer. The derivation of polymer dynamics near a wall in the semi-dilute regime also assumes that the 'blob' size (the dynamical coherence length of the monomers) grows like the distance to the wall [11]. As the width of the shear band in granular flows is always of the order of 10 bead diameters, regardless of the bead size, the bead diameter appears to be the only relevant length-scale in this problem. A linear increase of the coherence length with the distance to the wall is therefore the only reasonable choice 2 . For simplicity we will take exactly $r$ as the coherence length in the following.

Given this general picture, the scaling analysis follows: we define $N_{g}(r)$ as the number of particles involved in a crack developing at a distance $r$ :

$$
N_{g}=\left(\frac{r}{d_{g}}\right)^{D-1}
$$

The probability for this set of particles to become simultaneously unstable is $\left(p_{0}\right)^{N_{g}}$, whereas the time-scale $\tau(r)$ of the stress fluctuations experienced by the cluster of size $r$ reads:

$$
\frac{1}{\tau(r)}=\frac{V_{0}}{r}
$$

The scaling for $\tau(r)$ was made consistent with the criterion used for the first layer: $\tau(r)$ corresponds to a shear strain of 1 for the cluster of size $r$. Two different time-scales are thus involved in the dynamics of these blocks: a rapid one, $\tau_{0}$ associated with the release of the stress when a yielding occurs; a slower one, $\tau(r)$ which characterizes the stress fluctuations experienced by the particles at a distance $r$ from the wall.

Finally, a particle located at $y=r$ has $N_{g}$ possible locations along the crack and the expression for $P(r)$ eventually reads:

$$
P(r) d t \cdot \frac{d r}{d_{g}}=A \cdot N_{g} \cdot\left(p_{0}\right)^{N_{g}} \cdot \frac{V_{0} d t}{r} \cdot \frac{d r}{d_{g}}
$$

When yielding occurs at a distance $r$ from the wall, every particle below $y=r$ moves over a distance $d_{g}$ whereas no motion occurs above $y=r$. Such an event represents a discontinuity in the instantaneous

\footnotetext{
${ }^{2} \mathrm{~A}$ different mapping of the coherence length $l(r)$ may have to be used for a different geometry. For example, the stationary dense granular flow along an inclined plane seems to exhibit a characteristic length $H$ depending on the inclination and roughness of the inclined plane [12, 13]. We postpone the discussion of the coherence length in this case for a further study.
} 
velocity profile (the velocity is $V_{0}$ below the crack and 0 above during a time $\tau_{0}$ ). The constitutive differential equation for the stationary mean velocity profile $V(r)$ thus reads :

$$
\frac{\partial V}{\partial r}=-P(r)
$$

with the boundary conditions $V(0)=V_{0}$ and $V(\infty)=0$..

For $D=2$, we obtain:

$$
\frac{\partial V_{2 D}}{\partial r}=-\frac{A}{d_{g}} V_{0}\left(p_{0}\right)^{r / d_{g}}
$$

which by integration gives an exponential velocity profile $V_{2 D}(r)$ :

$$
V_{2 D}=V_{0} \cdot e^{-\frac{r}{\lambda d_{g}}} \quad \text { with } \quad \lambda=-\frac{1}{\ln \left(p_{0}\right)} \quad \text { and } \quad A=\frac{1}{\lambda}
$$

For $D=3$, it gives:

$$
\frac{\partial V_{3 D}}{\partial r}=-\frac{A r}{d_{g}^{2}} V_{0}\left(p_{0}\right)^{\left(r / d_{g}\right)^{2}}
$$

so that the velocity profile $V_{3 D}(r)$ is Gaussian:

$$
V_{3 D}=V_{0} \cdot e^{-\frac{r^{2}}{2\left(\sigma d_{g}\right)^{2}}} \quad \text { with } \quad \sigma^{2}=-\frac{1}{2 \ln \left(p_{0}\right)} \quad \text { and } \quad A=\frac{1}{\sigma^{2}}
$$

The function $P(r)$ then reads respectively in two and three dimensions:

$$
P_{2 D}(r, t)=\frac{V_{0}}{\lambda d_{g}} e^{-\frac{r}{\lambda d_{g}}} \quad \text { and } \quad P_{3 D}(r, t)=\frac{r \cdot V_{0}}{\sigma^{2} d_{g}^{2}} e^{-\frac{r^{2}}{2\left(\sigma d_{g}\right)^{2}}}
$$

Although a few hypothesis have been used to derive these results, one should note that the major assumption is that the persistence length of the fractures increases linearly with $r$. This argument alone produces the main behaviour of $P(r)$ as a function of $p_{0}$. The rescaling of the stress fluctuation time-scale $\tau(r)$ only controls the pre-factor of the exponential and Gaussian velocity profiles. A correction could also arise from a spatial dependence of $p_{0}$. For instance, the shear induced density profile neglected in the present approach might affect the uniformity of the average shear and normal stress per particle within the shear band. Considering that $p_{0}$ is a decreasing function of the density, this correction may increase the velocity decay and narrow the shear band. This may explain deviations from the Gaussian and exponential profiles observed in experiments with rounded and monodisperse particles where the largest density gradients are present. However, an exact solution of (3) for a general $p_{0}$ is highly non-trivial and should be investigated in a further work.

\section{FORCES DISTRIBUTION AND POWER SPECTRUM}

In addition to the velocity profiles, the knowledge of the function $P(r)$ gives appropriate information for computing other properties of the flow such as the probability distribution function (PDF) of the forces acting on the wall as well as the power spectrum of this signal. These two quantities have been experimentally studied in both two and three dimensional granular shear flows [5, 15]. In the latter experiment, the normal force on the bottom of a shear cell was monitored using a force transducer which could accommodate a large number (from 4 to 100) of particles. The resulting measurement corresponded to the integrated value of many individual contact forces. The measured force signal $\mathrm{F}(\mathrm{t})$ appeared as a series of narrow peaks of various heights. To compare these results with the present model, we suppose that each peak is the signature of a failure in the structure of the sheared pack. Assuming that the slippage of one grain releases a given force, the motion of a cluster of size $r$ produces a peak of intensity $F(r)$ proportional to $N_{g}$, or equivalently:

$$
F(r) \propto r^{D-1}
$$


These peaks last for a time $\tau_{0}$ and are uncorrelated in time. ¿From here, we can derive the form of the probability distribution function (PDF) $\rho(F)$ by identifying $r^{D-1}$ with $F$ in $P(r)$ :

$$
\rho_{2 D}(F)=\rho_{3 D}(F)=\frac{\exp \left(-F / F_{0}\right)}{F_{0}}
$$

where $F_{0}$ is the mean force of the distribution. Surprisingly enough, these PDF's are the same for 2and 3-D. Both agree with experimental observations at large forces [5]. The behaviour at low forces is not probed by experiments although it may have more complicated features.

One may note that eq. (4) is formally identical to what has been observed for large forces on individual particles in a compressed static array [16]. But this static distribution would narrow upon spatial averaging. By contrast, the width of the force distribution in the present description is insensitive to the integration area ${ }^{3}$ in agreement with experimental observations. In the continuously sheared regime, the wide force distribution arises from the coherence in the force release induced by the sudden motion of large clusters, and not from the purely static force distribution on individual particles.

The power spectrum of the force fluctuations can also be obtained in this description as follows: the power signal is decomposed into a stochastic succession of peaks of height $F^{2}(r)$ and width $\tau_{0}$. The period $\tau^{\prime}(r)$ between two successive pulses of amplitude $F^{2}(r)$ is simply the inverse of the rate $P(r)$. For a given $r$, and therefore a given $F(r)$, the signal is the so-called telegraph noise, for which the power spectrum $Q_{r}(\omega)$ reads [17]:

$$
\begin{gathered}
Q_{r}(\omega)=\frac{F^{2}(r)}{\pi} \frac{\tau^{\prime}(r) \tau_{0}}{\left(\tau^{\prime}(r)+\tau_{0}\right)^{2}} \cdot \frac{1 / T(r)}{\omega^{2}+(1 / T(r))^{2}} \\
\text { with } 1 / T(r)=1 / \tau^{\prime}(r)+1 / \tau_{0}
\end{gathered}
$$

Therefore, since failures at different distances from the wall are uncorrelated, the power spectrum $Q(\omega)$ for the overall signal is given by the sum:

$$
Q(\omega)=\frac{1}{d_{g}} \int_{0}^{\infty} Q_{r}(\omega) d r
$$

$Q(\omega)$ exhibits qualitatively the same behaviour for 2 and 3 dimensions, dominated by Lorentzian-like functions (see figure (2) ). For frequencies much larger than $\omega_{0}=1 / \tau_{0}$, the power spectrum behaves like $\omega_{0} / \omega^{2}$ as observed in experiments 115 ; this is a direct consequence of the stochastic description of the process. On the other hand, the expression (5) displays a well-defined non-zero limit as $\omega \rightarrow 0$. By comparison, the power spectra measured in [15] exhibit a continuous, although small increase as $\omega \rightarrow 0$.

\footnotetext{
${ }^{3}$ The finite size should merely show up as a cut of at high forces.
} 


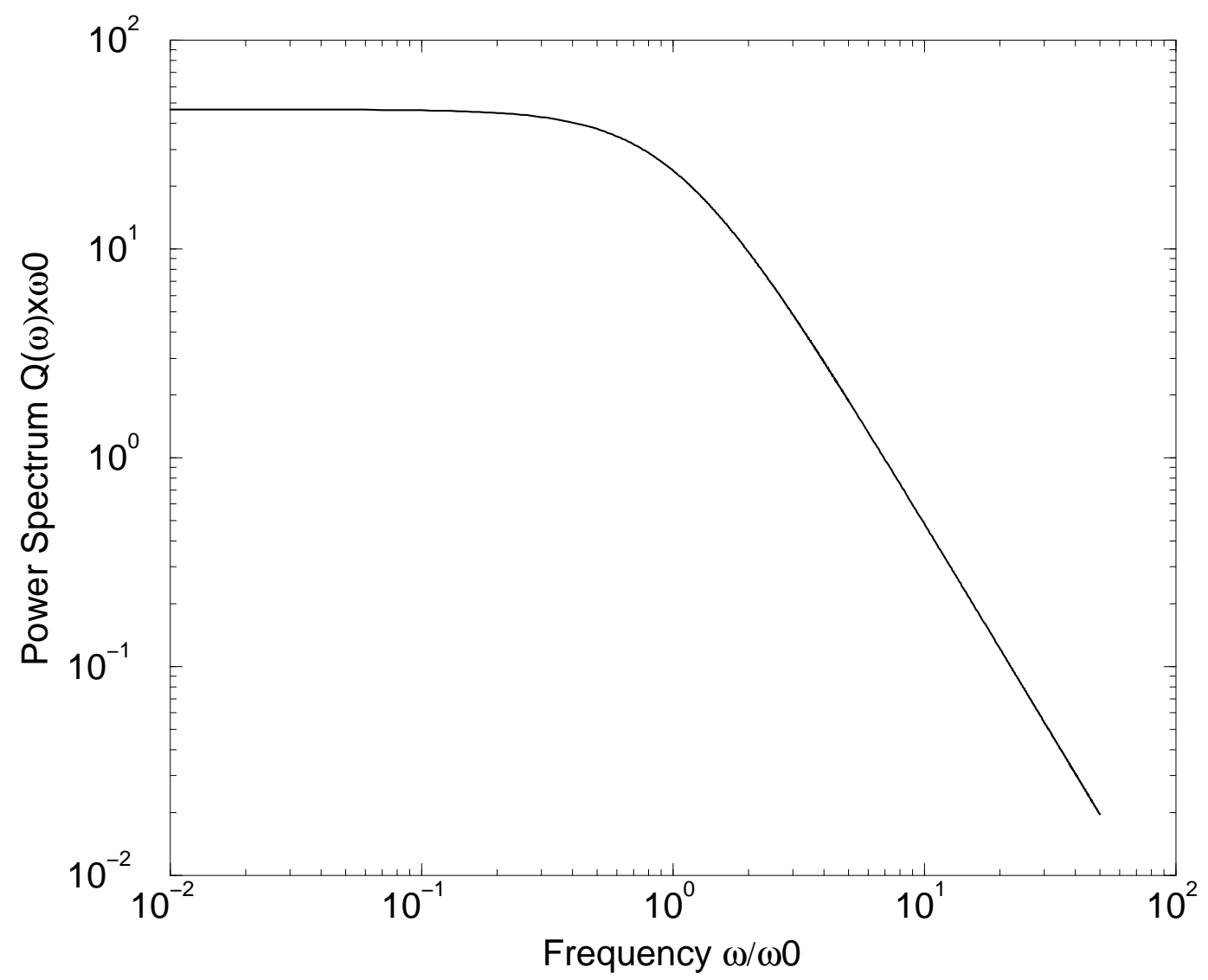

FIG. 2. Power spectrum of the forces in the $2 \mathrm{D}$ geometry. The curve has a flat part at low frequencies and a $1 / \omega^{2}$ behaviour for large frequencies. The cross-over between these two regimes occurs around the typical frequency $\omega_{0}$ for which the wall has moved by one grain diameter.

\section{CONCLUSION-ACKNOWLEDGEMENTS}

We have proposed a stochastic description of slowly sheared granular material that can capture the main characteristics of the flow: the average velocity profiles and force fluctuations in 2 and 3 spatial dimensions. We want to underline the relatively good agreement with experimental data with regards to the small number of ingredients introduced. In particular, we ignored the existence of granular density variations along the direction normal to the flow which may induce significant corrections. The crucial assumption of this model lies in the self-similar structure of moving clusters that rapidly form and disappear as the material flows. This assumption has been qualitatively suggested by the mere observation of 3-D Couette flows watched from below through a transparent bottom plate where the jerky and multi-scale dynamics is clearly visible. We hope that this tentative description will motivate further quantitative investigations to probe the nature of space and time correlations in the motion of neighboring particles in such systems.

Here we have focused on recent experimental data in order to test this self-similar model but we think that this simple description of dense granular dynamics as a super-imposition of coherent moves can be extended to many more geometries and processes than just plane shear. The issue is to correctly prescribe the coherence length mapping $l(r)$, and describe the probability $P(r)$ for each realization. In particular, we anticipate that the existence of multiple relaxation times recently demonstrated in granular compaction experiments [18] could be associated with rearrangements over different length-scales during the relaxation of the packing structure. To that extent, this model may offer a clue to understand the numerous features that granular material share with glassy liquids below the glass transition (aging, slow relaxation, jamming [19]).

It is a pleasure to thank D. Mueth, H. Jaeger, S. Nagel, T. Witten and L. Kadanoff for many interesting and stimulating discussions. G.D. is supported by the David Grainger Fellowship. C.J. is supported in part by the ONR grant: N00014-96-1-0127, the MRSEC with the National Science Foundation DMR grant: 9400379, and would also like to thank the Argonne National Laboratory for its support. 
[1] H. Jaeger, S. Nagel and R. Behringer, Rev. Mod. Phys. 68, 1259 (1996); J. Rajchenbach, in Physics of Dry Granular Media, NATO ASI Series E350, 421 (1998).

[2] C. K. K. Lun, S. B. Savage, D. J. Jeffrey and N. Chepurniy, J. Fluid Mech. 140, 223 (1984); P. Zamankhan, A. Mazouchi, and P. Sarkomaa, Appl. Phys. Lett. 71, 3790 (1997)

[3] S. Straub, Geol. Rundsch. 85, 85 (1996).

[4] For a review on continuous model, see R. Jackson, in Theory of Dispersed Multiphase Flow, edited by R. E. Mayer (Academic Press, New York, 1983), p 291.

[5] C. Veje, D. Howell, R. Behringer, S. Schöllmann, S. Luding and H. Herrmann, in Physics of Dry Granular Media, NATO ASI Series E350, 237 (1998); D. Howell, R. Behringer and C. Veje, "Stress Fluctuations in a 2D Granular Couette Experiment: A Continuous Transition.", preprint, submitted to Phys. Rev. Lett. (1999).

[6] D. M. Mueth, G. F. Debregeas, G. Karczmar, H. M. Jaeger and S.R. Nagel, preprint (1999).

[7] J.-P. Bouchaud, M.E. Cates, J. Ravi-Prakash, and S.F. Edwards, J. Phys. France I 4, 1383 (1994).

[8] C. Veje, D. Howell and R. Behringer, Phys. Rev. E, 59, 739 (1999). In fact, a tri-modal distribution is observed near the wall, at velocities $V_{0}, V_{0} / 2$ and 0 . The $V_{0} / 2$ is nothing but the velocity of a grain in rotation.

[9] O. Pouliquen and R. Gutfraind, Phys. Rev. E 53, 552 (1996).

[10] K. Glasstone, J. Laidler and H. Eyring, The theory of rate processes (Mc Graw-Hill, New-York, 1941).

[11] P-G De Gennes, "Scaling concepts in polymer physics", Cornell Univ. Press (1979).

[12] P. Mills, D. Loggia and M. Texier, Europhys. Lett. 45, 733 (1999).

[13] O. Pouliquen, preprint (2000).

[14] A.V. Tkachenko and V. Putkaradze, cond-mat/9912187 (1999).

[15] B. Miller, C. O’Hern and R. Behringer, Phys. Rev. Lett. 77, 3110 (1996).

[16] C. Liu, S. Nagel, D. Schecter, S. Coppersmith, S. Majumdar, O. Narayan and T. Witten, Science 269, 513 (1995); D.M. Mueth, H.M. Jaeger and S.R. Nagel, Phys. Rev. E 57, 3164 (1998).

[17] S. Machlup, J. Applied Phys. 25, 341 (1954).

[18] C. Josserand, A. Tkatchenko, D. Mueth and H. Jaeger, preprint (2000).

[19] A. J. Liu, S. R. Nagel, Nature 396, 21 (1998). 